www.jmscr.igmpublication.org

Impact Factor 5.84

Index Copernicus Value: 71.58

ISSN (e)-2347-176x ISSN (p) 2455-0450

crossref DOI: _https://dx.doi.org/10.18535/jmscr/v5i11.12

Journal Of Medical Science And Clinical Research

\title{
Clinical Correlation between McISSAC Score and Throat Swab Culture In Predicting Streptococcal Pharyngitis In Patients Presenting With Sore Throat
}

\author{
Authors \\ S.Usha $^{* 1}$, Ruta Shanmugam ${ }^{2}$, R.Prem Nivas ${ }^{3}$, V.U.Shanmugam ${ }^{4}$, R.G.Mariappan ${ }^{5}$, \\ Balaji Swaminathan $^{6}$, Bharath ${ }^{7}$ \\ ${ }^{*}$ Post Graduate, Department of ENT, RMMCH, Chidambaram-608002, Tamilnadu \\ ${ }^{2}$ HOD, Department of ENT, RMMCH, Chidambaram-608002, Tamilnadu \\ ${ }^{3}$ Assistant Professor, Department of ENT, RMMCH, Chidambaram-608002, Tamilnadu \\ ${ }^{4,5}$ Professor, Department of ENT, RMMCH, Chidambaram-608002, Tamilnadu \\ ${ }^{6}$ Reader, Department of ENT, RMMCH, Chidambaram-608002, Tamilnadu \\ ${ }^{7}$ Senior Resident, Department of ENT, RMMCH, Chidambaram-608002, Tamilnadu
}

\begin{abstract}
Introduction: GAS pharyngitis is both antecedent for invasive streptococcal infection and post infectious immunologic complication of Rheumatic fever/RHD, a leading cause of cardiovascular morbidity and mortality in many developing parts of the world.
\end{abstract}

Aims and Objective: To analyse the usefulness of symptoms and signs in assessing the risk of streptococal pharygitis and to correlate McISSAC score with throat swab culture.

Results: About 50\% of patient tested positive for GABHS have Mc-Issac score of 5, whereas $25 \%$ of positive tested patient have score of 4 and again $25 \%$ of positive patient have score of 3 . The chi-square test of association is significant. Therefore, $75 \%$ of positive GABHS findings have Mc-Issac score of 4 and 5.

Conclusion: Individual signs and symptoms are not powerful enough to discriminate GABHS pharyngitis from other types of sore throat. The McIsaac score is well calibrated clinical prediction rule for estimating the probability of GABHS pharyngitis. The result of McIsaac score and culture finding of GABHS have close association with each our.

Keywords: McIsaac score, GABHS, Throat swab culture and sensitivity.

\section{Introduction}

Sore throat is a common complaint in upper respiratory tract infection and may indicate infection with Group A streptococci. ${ }^{1}$ Although viruses cause most acute pharygitis episodes, Group A streptococci causes $37 \%$ of cases of acute pharyngitis in children older than 3 years. GAS pharyngitis is both antecedent for invasive streptococcal infection and post infectious immunologic complication of Rheumatic fever/RHD, a leading cause of cardiovascular morbidity and mortality in many developing parts of the world.

McIssac developed four criteria to predict the probability of the presence of streptococcus pyogenes in a throat swab culture and thereby 


\section{JMSCR Vol||05||Issue||11||Page 29876-29880||November}

appropriately prescribe antibiotics to alleviate symptoms and decrease the rates of acute Rheumatic fever, suppurative complications, missed school and work days ${ }^{1}$

Throat swab culture and sensitivity is considered to be the reference standard for the diagnosis of streptococcal pharyngitis. Clinical prediction rules have been developed over the last 40yrs to distinguish streptococcal pharyngitis from other causes. The most widely recognised Clinical prediction rule for GABHS pharygitis is the McISSAC score.

\section{Aims and Objective}

To analyse the usefulness of symptoms and signs in assessing the risk of streptococal pharygitis and to correlate McISSAC score with throat swab culture

\section{Material and Methods}

Study was conducted on a total of 100 patients who presented to ENT Department at Rajah Muthiah Medical College Hospital, Chidambaram during OCTOBER 2016 TO SEPTEMBER
2017.Patients aged 3years and above and all patients who presented with sore throat were included in the study. Children below 3years, patients on antibiotic therapy in the previous week, Immuno compromised patients were excluded from the study. Patients included in the study were scored as per McIsaac score.

\begin{tabular}{|l|l|}
\hline Clinical criteria & Points \\
\hline Absence of cough & 1 \\
\hline Swollen and tender nodes & 1 \\
\hline Temperature $>38 \mathrm{c}$ & 1 \\
\hline Tonsillar exudates or swelling & 1 \\
\hline Age 3 to 14 years & 1 \\
\hline Age 15 to 44 years & 0 \\
\hline Age 45 years and older & -1 \\
\hline
\end{tabular}

Throat swab was taken for all these patients. McIsaac score and Throat swab culture and sensitivity results were compared and analysed statistically.

\section{Results}

The mean age of our patients was $22.59 \pm 12.06$ years. The majority of patients were female $(56.9 \%)$

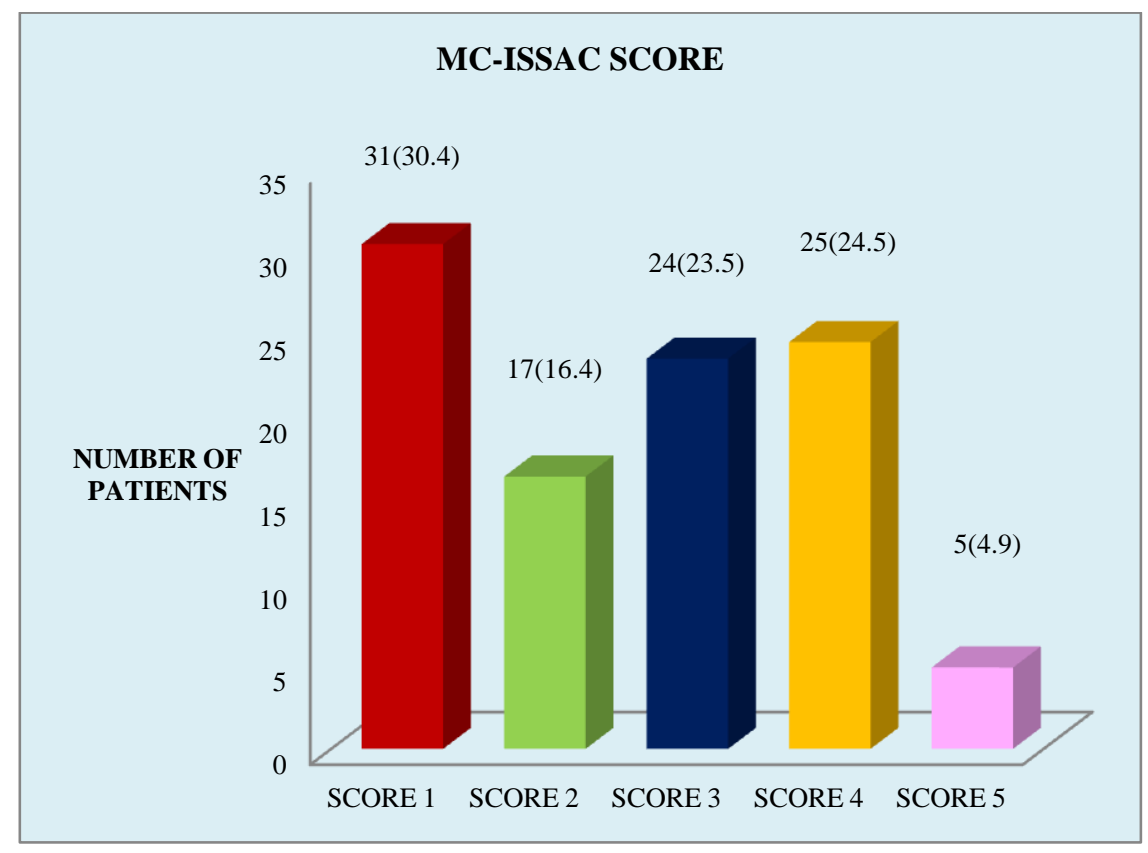

Out of 102 patients, 49 patients $(49 \%)$ had McIsaac score of $3 \& 4$. 
Table-1: Association of Mc-ISSAC score with GABHS

\begin{tabular}{|c|c|c|c|c|c|c|c|c|}
\hline \multirow{3}{*}{$\begin{array}{l}\text { Mc- } \\
\text { Issac } \\
\text { Score }\end{array}$} & \multicolumn{6}{|c|}{ GABHS } & \multicolumn{2}{|c|}{ Chi-Square Test } \\
\hline & \multicolumn{2}{|c|}{ Positive } & \multicolumn{2}{|c|}{ Negative } & \multicolumn{2}{|c|}{ Total } & Value & Significant \\
\hline & $\mathbf{N}$ & $\%$ & $\mathbf{N}$ & $\%$ & $\mathbf{N}$ & $\%$ & \multirow{6}{*}{19.24} & \multirow{7}{*}{.001} \\
\hline 1 & - & - & 31 & 31.6 & 31 & 30.4 & & \\
\hline 2 & - & - & 17 & 17.3 & 17 & 16.7 & & \\
\hline 3 & 1 & 25 & 23 & 23.5 & 24 & 23.5 & & \\
\hline 4 & 1 & 25 & 24 & 24.5 & 25 & 24.5 & & \\
\hline 5 & 2 & 50 & 3 & 3.1 & 5 & 4.9 & & \\
\hline Total & 4 & 100 & 98 & 100 & 102 & 100 & & \\
\hline
\end{tabular}

Out of 54 patients with McIsaac score of 3 to 5, GABHS was cultured on throat swab in 4 patients.However, in patients with McIsaac sore of $1 \& 2$,Gabhs was not found on throat swab culture.
The chi-square test of association was statistically significant $\left(\mathrm{X}^{2}=19.24, \mathrm{P}=.001\right)$. Therefore GABHS finding is significantly associated with Mc-Issac score.

Table - 2: Comparison of GABHS VS MC-Issac Score by sensitivity and specificity analysis

\begin{tabular}{|l|c|c|c|}
\hline GABHS & Positive & Negative & Total \\
\hline Positive & True Positive $=3$ & False Positive $=27$ & Total Positive $=30$ \\
\hline Negative & False Negative $=1$ & True Negative $=71$ & Total Negative $=72$ \\
\hline & T.Positive $=4$ & T.Negative $=98$ & 102 \\
\hline
\end{tabular}

Table -3: Outcome of Comparison

\begin{tabular}{|c|c|}
\hline Measure & Percentage \\
\hline Sensitivity = $\begin{array}{c}\text { T.P } \\
\text { T.P+F.N } \\
\text { T.----- X } 100\end{array}$ & $75 \%$ \\
\hline 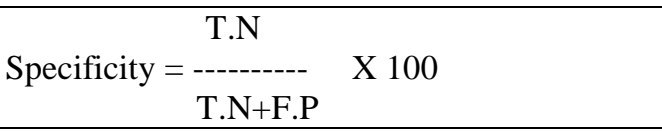 & $72.45 \%$ \\
\hline 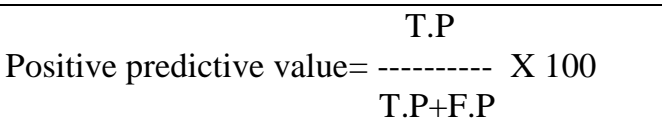 & $10 \%$ \\
\hline 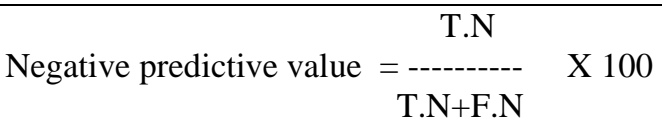 & $98.61 \%$ \\
\hline
\end{tabular}

The sensitivity is $75 \%$, which means that Mc-Issac score is rightly identifying the disease in $75 \%$ of the patients. The specificity is $72.45 \%$, which means that Mc-Issac score is rightly identifying no disease in $72.45 \%$ of the patients. The positive predicted value is only $10 \%$, that is if the test result is positive, the probability of having disease is only $10 \%$. The negative predicted value is high ie $98.61 \%$. If the test result is negative, the probability of not getting the disease is $98.61 \%$.

\section{Discussion}

GABHS is accepted to be the most important pathogenic bacterium that causes sore throat because of its sequela and complications Tonsillopharytngitis due to streptococci is a potentially serious disease as it cancause suppurative complications like rheumatoid valvular heart disease and acute glomerulonephritis. As a result, rapid diagnosis and adequate treatment are necessary. For 50 years, the fundamental test has been throat swab culture. It detects $90 \%-99 \%$ of positive cases and is accepted as the gold standard in GABHS diagnosis. 
Studies comparing clinical diagnosis with throat culture have shown a sensitivity of $50 \%$ to $70 \%$ and a specificity of $60 \%$ to $80 \%$.Thus clinical judgement may miss up to $50 \%$ of GAS infections while identifying $20 \%$ to $40 \%$ of the large number of non-GAS sore throat presentations. Clinical prediction rules have been proposed as a way to increase the accuracy of clinical diagnosis. Although several scores have been proposed for assessing patients with sore throat, Centor \& McIsaac scoring system are most widely used clinical prediction rules.

In our study McIsaac scoring system had sensitivity $75 \%$. Specificity $72.45 \%$, positive predictive value $10 \%$, negative predictive value $98.61 \%$.

Amber Hanif Palla studied 137 patients using McIsaac scoring system. McIsaac scores were found to be $100 \%$ sensitive and $68.7 \%$ specific in their study, giving a positive predictive value (PPV) of $12.7 \%$ and a negative predictive value (NPV) of $100 \%{ }^{3}$

Ching-Tang Shih et al studied 342 patients using McIsaac scoring system and throat swab cultures. A McIsaac score of 5 had a sensitivity of $71 \%$, specificity of $70 \%$, and a positive predictive value of only $9.3 \%{ }^{4}$

The McIsaac scoring system is a simple primary care management approach that improves identification of GAS infection, limits the need for throat swabs in all patients with sore throat. ${ }^{1}$

\section{Conclusion}

Individual signs and symptoms are not powerful enough to discriminate GABHS pharyngitis from other types of sore throat. The McIsaac score is well calibrated clinical prediction rule for estimating the probability of GABHS pharyngitis. The result of McIsaac score and culture finding of GABHS have close association with each our.

\section{Bibliography}

1. W J McIsaac, D White, D Tannenbaum, and D E Low A clinical score to reduce unnecessary antibiotic use in patients with sore throat CMAJ. 1998 Jan 13; 158(1): 75-83.

2. Ana Gabriela P. dos Santos; Eitan N. Berezin Comparative analysis of clinical and laboratory methods for diagnosing streptococcal sore throat J. Pediatr. (Rio J.) vol.81 no.1 Porto Alegre Jan./Feb. 2005

3. Amber Hanif Palla, Rafeeq Alam Khan, Anwar H. Gilani Overprescription of antibiotics for adult pharyngitisis prevalentin developing countries but can be reduced using McIsaac modification of Centor scores: a cross-sectional study

4. Ching-TangShih Ching-ChiangLin ChungChingLu Evaluation of a Streptococcal Pharyngitis Score in Southern Taiwan Volume 53, Issue 1, February 2012, Pages 49-54.

5. Yavuz FURUNCUOĞLU1, Filiz SAĞLAM, Ahmet KUTLUHAN3 Acute exudative tonsillitis in adults: the use of the Centor score and some laboratory testsTurk J Med Sci (2016) 46: 1755-1759 (c) TÜBİTAK doi: 10.3906/sag-1510-93.

6. Imanuel Y. Malino, Dwi Lingga Utama, Yati Soenarto McIsaac criteria for diagnosis of acute group-A $\beta$-hemolytic streptococcal pharyngitis VOLUME53 September 2013 Number 5

7. ElenaChiappini MD, PhD Nicola PrincipiMD, Italian panel on the Management of pharyngitis in children.Management of Acute Pharyngitis in Children: Summary of the Italian National Institute of Health Guidelines, Volume 34, Issue 6, June 2012, Pages 1442-1458.e2.

8. Richelle J. Cooper, MD, MSHS; Jerome R. Hoffman, MA, MD; John G. Bartlett, MD; Principles of Appropriate Antibiotic Use for Acute Pharyngitis in Adults: Background POSITION PAPERS $\mid 20$ MARCH 2001

9. BETH A. CHOBY, MD, University of Tennessee College of Medicine-Chattan- 
ooga, Chattanooga, Tennessee Diagnosis

and Treatment of Streptococcal

Pharyngitis Am Fam Physician.

2009 Mar 1; 79(5):383-390.

10. Susanna Felsenstein, Diala Faddoul,

Richard Sposto, Molecular and Clinical

Diagnosis of Group A Streptococcal

Pharyngitis in Children J Clin Microbiol.

2014 Nov; 52(11): 3884-3889.

doi: 10.1128/JCM.01489-14

11. Tony MO Bakare and Peter Schattner. The usefulness of a clinical 'scorecard' in managing patients with sore throat in general practice Asia Pac Fam Med. 2010; 9(1): 9. Published online 2010 Jul 29. doi: 10.1186/1447-056X-9-9. 